\title{
Hemodynamic Status and Its Relationship with the Risk of Pressure Ulcers Development in Patients After Open Heart Surgery
}

\author{
Mojtaba Senmar ${ }^{1}$; Hossein Aliakbari ${ }^{1}$; Jalil Azimian ${ }^{2}$; \\ Hossein Rafiei ${ }^{3}$; Azam Mohammadi Alamouti ${ }^{4}$ \\ ${ }^{1}$ MSc in Critical Care Nursing, Department of Nursing, School of Nursing and Midwifery, \\ Qazvin University of Medical Sciences, Qazvin, Iran \\ ${ }^{2}$ Assistant professor, Department of Nursing, School of Nursing and Midwifery, \\ Qazvin University of Medical Sciences, Qazvin, Iran \\ ${ }^{3}$ Instructor, Department of Nursing, School of Nursing and Midwifery, \\ Qazvin University of Medical Sciences, Qazvin, Iran \\ ${ }^{4}$ Instructor, Department of Nursing, School of Nursing and Midwifery, \\ Islamic Azad University- Karaj branch, Alborz, Iran \\ Correspondence to: Jalil Azimian.Email:azzimianj@yahoo.com
}

\begin{abstract}
Objective: Despite using the most appropriate methods of prevention, the incidence of pressure ulcers is still considered as a major problem of health care in all health systems. A group of patients at high risk for this problem, there are patients who are undergoing open heart surgery. But few studies have been done about the risk of pressure ulcers in this group of patients. This study aimed to investigate the relationship between hemodynamic statuses with the risk of pressure ulcers development in patients after heart surgery is done.

Materials and methods: This cross-sectional study conducted in cardiac intensive care unit of Bu Ali Sina hospital in Qazvin. Eighty two patients who were undergoing cardiac surgery were selected by convenience sampling method. The risk of pressure ulcers on three times, before surgery, after surgery (the patient's consciousness) and at discharge was evaluated by using the Braden scale and international staging pressure ulcers (stage 6). As well as demographic specifications, hemodynamic status, including systolic blood pressure, diastolic blood pressure, mean arterial blood pressure, heart rate, central venous pressure and the patient's medications on a daily basis were recorded by the researcher. Data analysis was performed using SPSS 16 software.
\end{abstract}

Results: Of the 82 patients, 36 were women (43.9\%) and 46 males (56.1\%). The mean ages of patients were $10.52 \pm 60.93$ and the mean of intensive care unit stay was $2.87 \pm 0.96$. The risk of pressure ulcers with an average diastolic blood pressure on the first day $(p=0.04)$, the mean central venous blood pressure in the second day $(p=0.01)$, mean heart rate on the second day $(p=0.04)$, age $(p=0.000)$, duration of hospitalization in a special section $(p=0.002)$, presence of diabetes $(p=0.04)$, cardiac surgery $(p=0.01)$, smoking $(p=0.000)$ and the pump during surgery $(p=0.03)$ had a significant relationship.

Conclusion: according to finding of present study, patients who are undergoing cardiac surgery, are at high risk for pressure ulcers development and factors such as diastolic blood pressure, central venous pressure, increased heart rate, increased age, duration of hospitalization, presence of diabetes, previous surgery, smoking and working the pump during surgery could be increased this risk.

Keywords: pressure ulcer, hemodynamic, cardiac surgery, risk, manifestation

\section{Introduction}

Pressure ulcer, pressure sore or decubitus ulcer (1) is localized damage to the skin or underlying tissue usually over a bony prominence (2). Local with soft tissue less (3) with at least 5,000 years of history (4), Health care remains a major challenge with the prevalence of almost $25 \%$ in the United States of America and between 10 to 50 percent in Iran (5). Many research has done in the field of identifying factors pressure ulcers in nursing profession and nurses always in providing nursing services has encountered to patients that in the initial review are susceptible to pressure ulcers (6). Many factors affect the development and progression of pressure ulcers. (7) and a large number of risk factors have been identified for development of pressure ulcers (8), because of pressure ulcer is a multifactorial problem (9). That some of these environmental factors and some were related to one's own (7) and as well as primary risk factors. (10). Including external factors pressure and friction (11) and internal factors hematologic criteria. (12), malnourishment. (11), hemodynamic changes. 
(9), blood glucose levels (9), and blood glucose (9). However, only one factor can explain the incidence of pressure ulcers (12). Causes of pressure ulcers, in recent years, in the world hospitals, is one of the concerns of medical staff, especially nurses (13). In the meantime, the primary responsibility for the prevention of pressure ulcers is a nursing (14). For this reason, the prevention of pressure ulcers is one of the oldest methods of prevention in nursing. (15). In addition, the key factor of prevention of pressure ulcers is an in quality care. (16), because pressure ulcers is still one of the important issues in the care of patients. (17). Accordingly, due to the many problems associated with the prevention of pressure sores, the preventing of development of them, is one of the most important issues in the scientific literature. (18). Meanwhile, there many situations like hospitalization in intensive care units and performed surgical procedures on patients that is putting the patient expose to and development and progression of pressure ulcer.

Patients undergoing surgery due to sensory impairment, immobility disorder ,hemodynamic changes and the use of vasoconstrictors drugs during surgery are at increased risk of developing pressure ulcers (20).Given that the ICU patients spend a lot of time in bed, and taking into account the dramatic changes cardiovascular system - dependent vascular bed rest (21), These patients are always at risk of hemodynamic disorders (22). On one hand, hemodynamic in patients after cardiac surgery despite the success of surgery is often unstable (23). And the defects in the overall blood circulation will increase the risk of pressure ulcers (10). Statistics indicate the incidence of pressure ulcers between 17 and 29.5 percent in patients following cardiac surgery (24). And in Iran, the amount of incidence of pressure ulcers after heart surgery has been reported in $3 / 21$ percent (24). In addition, patient's cardiovascular surgery is responsible for almost 45 percent of total hospital costs to treat pressure ulcers (24). Apart from these costs, the incidence pressure ulcer is concerned with pain (2), increased risk of osteomyelitis, endocarditis (13), increasing the workload of the health care system (24) and death (13).

Based on the evaluate of previous studies, at the moment, enough evidences about the relationship between hemodynamic status with the incidence risk of pressure ulcers in patients undergoing cardiac surgery is not entirely clear. So, in this regard and given the importance of the issue, this study aimed to investigate the relationship between hemodynamic statuses with the incidence risk of pressure ulcers in patients after open heart surgery was performed.

\section{Method}

This descriptive- analytical in kind of cross-sectional was conducted in ICU unit of BU- Ali Sina hospitals heart surgery dependent on the Medical Sciences University and Health Services of Qazvin in 1395. Necessary permission for the study was obtained from the University of Medical Sciences. Also before the start of the study, necessary explanations were given the examples and of their satisfaction of participation in the study was obtained. Also participating in the study sample was assured that the information obtained from them will remain confidential and will be used only for the purposes of the study. The aim population of this study included all patients who had open heart surgery and were admitted in this section. This includes 6 beds and 14 staff nurses. Sampling in this study, was convenience method and include all patients who inclusion criteria and exclusion criteria of the study were applied to them. Inclusion criteria to study, tendency to participate in the study with informed consent, age over 18 years and was the lack of pressure ulcers on arrival. Patients who require emergency surgery, and there was no opportunity to review their skin before surgery, were excluded. Total sample size was 66 respondents who expect to lose 10 to 20 percent, 82 patients were enrolled in the study. Third-party data collection tool, the first part includes demographic information List such as age, sex, history of developing pressure ulcers, pressure ulcers degree, history of previous diseases, the cause of exclusion of study, exit date of patients from the cardiac surgery departure, the body mass index, pump status during surgery and obtained scores of the Braden tool. The second part of the tool, including the check list collected clinical information, including information related to hemodynamic (systolic blood pressure, diastolic blood pressure, mean arterial blood pressure, heart rate, central venous pressure), respectively. The third part of Braden tools, was predictor tool of the incidence risk of pressure ulcers. This criterion includes 6 parameters: sensory perception, moisture, activity, mobility, nutrition and force wear. Each of these parameters with the exception of rubbing force from 1 to 4 and the force of friction is 1 to 3 points. The score 1 shows the worse situation and 4 shows the better situation. At the end, after collecting any of the scores, the final score was obtained. That the lower score indicates a risk situation. Also, the maximum score is 23 . It should be noted that Braden tool used in the study Montazeri et al. And the reliability of this criterion is $83 \%$ and sensitivity and specificity of it equal to $92 \%$ and $74 \%$ respectively (25). At the beginning of every day, after receiving the surgeries list of this section, When the patient entered the operating room door, areas susceptible to pressure ulcers in each patient thoroughly examined and evaluated. Braden tool was used first before entering the operating room. If have pressure sores when entering the operating room, according to patient entry criteria were excluded from the study. 
Check List of patient demographic specifications such as age, sex, date of admission, disease history, diabetes illness, hypertension, hyperlipidemia, previous stroke, previous surgical history, body mass index was recorded at the time of entering the operating room. After emersion the patient from the operating room, pump status during surgery of the case and the questions of the doctor was completed. Wavy mats were recorded too. For the second time, after removal of the patient from the operating room Braden tool was applied. Prone areas for the occurrence of pressure ulcers was evaluated and if there is pressure ulcer according to international staging the pressure ulcer (stage 6) the wound level was recorded. Study of patient's skin, until discharge from the section, has been done on a daily basis and in If the improvement or development of new lesions was recorded in the corresponding file to patient.

Information related to the variables and the hemodynamic parameters on a daily basis based on the average daily and routine and rules were recorded. Braden tool was used for the third time in discharge time.

Data analysis using SPSS software and with the help of descriptive tests such as the number and percentage for qualitative variables (having a pressure ulcer, having diabetes, previous surgery, hypertension, history of stroke, hyperlipidemia, sex) and the mean and standard deviation for quantitative variables (age, length of stay, days of developing pressure ulcers, sore healing day, Braden tool score, body mass index, systolic blood pressure, diastolic blood pressure, mean arterial blood pressure, heart rate heart, blood pressure, central venous pressure) were studied. In order to examine the study variables, in two modes of variables from independent t-test to evaluate the relationship between independent variables with dependent variable and the was used Pearson correlation coefficient.

\section{Results}

During this study, one patient was excluded because of the urgency and one patient was excluded from study because of death. All patients for participating in the study have the necessary cooperation and none of the patients because of lack of consent to participate in the study were excluded. Of the 82 patients, 36 women $(43.9 \%)$ and 46 males $(56.1 \%)$. The average age of patients, was $10.52 \pm 60.93$ and the average days of hospitalization in a special section of heart surgery was $0.96 \pm 2.87$ days. Of the 82 patients admitted to the Special Section of Cardiac Surgery 27 (32.9\%) patients had pressure sores grade one. 4 (4.9\%) of patients had grade II pressure ulcer. Of the 27 patients with pressure ulcers, 25 persons on the first day got pressure ulcers grade one and two people in the second day of hospitalized were with wounds of pressure ulcers grade two. $\mathrm{T}$ test results showed that there was no significant correlation between the incidence of pressure ulcers with age $(\mathrm{P}=0.000)$, and the hospitalization time in section $(\mathrm{P}=0.002)$. In addition, chi-square test results showed that the presence of diabetes $(\mathrm{P}=0.04)$, previous surgery $(\mathrm{P}=0.01)$ and the pump situation during surgery $(\mathrm{P}=0.03)$ has a statistically significant relationship with the occurrence of pressure ulcers. In the meantime, patients who had pressure ulcers, than those without pressure ulcers older and had more length of stay on section. In the pump status variable analysis showed that all 27 patients with pressure ulcers have the "on" pump during surgery.

The findings of the study showed that between the incidence of pressure ulcers with the mean systolic blood pressure and mean arterial on the first, second, third and fourth days of hospitalization, there is no significant relationship. The findings also showed that between the incidence of pressure ulcers with an average diastolic blood pressure during the second, third and fourth days, there was no significant correlation.

\section{Discussion}

Although if appropriate interventions almost 95 percent of all pressure ulcers are preventable (24), however, pressure ulcers may cause serious complications (5). This study aimed to examine the association between hemodynamic variables and the risk of incidence of pressure ulcers in patients undergoing open heart surgery. The results of current study showed that the patients were at high risk of pressure sores and factors such as reduced diastolic blood pressure, central venous blood pressure and increased heart rate are effective in this matter. The results of current study showed that there is no significant relationship between the incidence of pressure ulcers with mean systolic blood pressure in the days of hospitalization. However, the association was not statistically significant, but, mean systolic blood pressure in the group has a wound on the first, second and fourth hospitalization days, was higher than the group without ulcers. In the study of Ghavidel and Et al., that was conducted to determine the degree of incidence and predisposing factors of pressure sores after open heart surgery; was found that blood pressure less than $80 \mathrm{~mm} \mathrm{Hg}$ in the incidence of ulcers was effective (24). In this study, only periods of reduced blood pressure is evaluated and differences in the impact of other variables on the basis of days has not been studied in the incidence of pressure ulcers and that's why the effect of parameters of this study is different with our study. It also in the study of Ghavidel, information within the operating room is examined. And surgery room conditions are very different than the section, because, direct manipulation of the hemodynamic System of inside the operating room takes place and further changes in systolic blood pressure occurs. While the Ghavidel and Et al., believe that systolic blood pressure less than $80 \mathrm{mmHg}$ are effective that in our study, daily average systolic pressure is not reached to less than $80 \mathrm{~mm} \mathrm{Hg}$. In the study of Shiu-piu and 
et al., found that periods of low blood pressure is associated with the incidence of pressure ulcers (26). This finding was contrary to the findings of our study, because in our study the mean systolic blood pressure in patients is not reached to less than $110 \mathrm{~mm} \mathrm{Hg}$. In this study, systolic blood pressure less than $90 \mathrm{mmHg}$ is considered, and it is clear that, when systolic blood pressure reach to less than $90 \mathrm{~mm} \mathrm{Hg}$ more blood flow to vital organs and skin usually exposed to the damage. In the study of Fallahi Nia and et al., found that there is no statistically significant relationship between the incidence of pressure ulcers with systolic blood pressure, (27). That this finding is consistent with the findings of our study. The results of current study showed that there was no significant correlation between the incidence of pressure ulcers with diastolic blood pressure during the second, third and fourth days of hospitalization and the incidence of pressure ulcers with diastolic blood pressure on the first day of hospitalization has a significant relationship. In the study of Santooran and et al., found that patients with pressure ulcers, have lower diastolic pressure (14). In our study, the incidence of pressure ulcers is a significant association with diastolic blood pressure on the first day. Of course, patients with pressure have experienced lower ulcers diastolic blood pressure in the first day. Santoorans study results are consistent with the results of our Study on the first day. Because the incidence of pressure ulcers in the first day has a significant relationship with diastolic blood pressure. Although diastolic blood pressure of patients of our study in the first days to three was lower in the patients with pressure ulcers, but diastolic blood pressure in patients with pressure ulcers in four days of hospitalization is higher. The difference in results can be attributed to differences in methods. Because the Santooran and Et al have started collecting their data when 24 hours of mechanical ventilation have been passed. Meanwhile, we have to analyze our results based on the day of admission. And Santooran and et al., have described the results as a whole. Belle and Et al study also showed that the incidence of pressure ulcers are associated with lower diastolic blood pressure (28), that these results are consistent with the results of our study on the first day of hospitalization. Belle et al., have expressed their results in general, while their patients have resided in hospital on average 24 days. Thus, if they mentioned it base on the day of admission interpret the results were better done. However, the results on the first day are consistent with the results of Belle et al., and is different in the next days. The results of this study showed that there is no significant relationship between the incidence of pressure ulcers with an average of arterial blood pressure in the first, second, third and fourth days of hospitalization. In the Study of Tryksy et al., found that the incidence of pressure ulcers is a significant correlation with mean arterial pressure (29). The results of this study are inconsistent with the results of our study. The difference in results can be attributed to the length of stay for patients with pressure ulcers, because of the length of stay for patients with pressure ulcers in Tryksy et al., study was close to 19 days, and while in our study duration was nearly three days. On the other hand, patients in our study had an average pressure lower than $85 \mathrm{~mm} \mathrm{Hg}$, while in the study of Tryksy et al., Mean arterial pressure of patients, on average was $55 \mathrm{~mm} \mathrm{Hg}$. Hence, the disaffiliation incidence of pressure ulcer with mean arterial pressure (MAP) is the justify in our study. In the study of Pender and Fries became clear that, contrary to our study there is a significant correlation between mean arterial blood pressure and the incidence of pressure ulcers (30). Differences in the statistics may be linked to differences in. Because the average of hospitalization days of patients in the study Pendrz and Fries was 13.5 days and in our study is nearly three days. On the other hand, mean arterial pressure in study of Penderz and Fries is much lower than ideal conditions. While in our study, the average mean arterial pressure is greater than ideal conditions or are within the scope of ideal. For this reason, disaffiliation of the incidence of pressure ulcers with mean arterial pressure in our study is explainable. In a study of Kadygan et al., found that the incidence of pressure ulcers has no significant correlation with mean arterial pressure (31), the results of this study are consistent with the results of our study. The results of our study showed that incidence of pressure ulcer with mean central venous blood pressure and heart rate on the second days of hospitalization has a significant relationship. Ghaseminejad et al., study found that there is no relationship between the incidences of pressure ulcers with heart rate (22). Lack of association between this variable and the incidence of pressure ulcers can be attributed to the length of hospitalization in patients with pressure ulcers and conditions of them in hospitalization days of this study. Because in study of Ghaseminejad et al., the patients were hospitalized an average of 14 days. However, the average of Hospitalization days of patients in our study were nearly three days. And patients in cardiac surgery experience most changes in hemodynamic than other patients. At any time based on days likely changes in heart rate is proposed. While Ghaseminejad et al., have suggested their results as a whole and not on days. In the study of Penderz and Friez found that the development of pressure ulcers is not significantly associated with heart rate (30). The results of this study are consistent with the results of our study on the first, third and fourth of hospitalization day. And lack of consistency on the second day, probably can be attributed to possible changes in the patient's clinical status. In the results of our study, the incidence of pressure ulcers with the mean central venous blood pressure, has a significant relationship in the second day and patients with pressure ulcers on the first day and the second day have higher central venous blood pressure. 


\section{Conclusion}

Results of this study showed that patients with open heart surgery, as well as other special sections are exposing to greater risk of incident of pressure ulcers, and factors such as reduction in diastolic blood pressure, central venous blood pressure and heart rate are influence in the increase of incidence of pressure ulcers. This issue suggests that those involved in the care and treatment of patients, especially nurses should have more attention to the prevention of pressure ulcers and with a comprehensive review whether in terms of patient physical examination and whether in terms of review of medical history are prevented from incidence of Pressure ulcers. And pay more attention to the patient due to wide variation in the hemodynamic parameters during their hospitalization in relation to the incidence of pressure ulcers.

The results of this study can be used in the Educational sections of University faculties retraining courses and workshops within the hospital and sooner identify of patients at risk of incidence of pressure ulcers be used by health care workers. Of the strengths of this study using highest effective variables in occurrence of the pressure ulcer, using observation method in the patient's bedside and case records can be named. About the research limitations, the quality of nursing care, is one of the factors that has a major effect in creation of pressure ulcer that has not been investigated in this study. It is recommended that a study simultaneously; review inside conditions and variables, and after the operating room. Until more detailed information of the factors that influence the incidence of pressure ulcers is achieved. Finally, our gratitude of all those who somehow helped the research team in the research, especially the staff and hospitalized patients at Special section of Bu- Ali sina Hospital in open heart surgery.

\section{Refrences}

[1]. Ruggiero C. Pressure Ulcer Assessment and Documentation. Master's Theses, Dissertations, Graduate Research and Major Papers Overview. Paper 127. 2015.

[2]. Chaboyer W, Mills PM, Roberts S, Latimer S. Physical activity levels and torso orientations of hospitalized patients at risk of developing a pressure injury: An observational study. International Journal of Nursing Practice. 2015; 21(1): 11-7.

[3]. McGinnis E, Greenwood DC, Nelson EA, Nixon J. A prospective cohort study of prognostic factors for the healing of heel pressure ulcers. Age and ageing. 2013: aft187.

[4]. Rubayi S. Pressure Ulcers: An Important Condition in Medicine and Surgery. Reconstructive Plastic Surgery of Pressure Ulcers. Berlin, Heidelberg: Springer Berlin Heidelberg. 2015. 1-4.

[5]. Azimian J, Nayeri Dehghan N, Pourkhaleghi E, Ansari M. Transdermal Wound Oxygen Therapy on Pressure Ulcer Healing: A Single-Blind Multi-Center Randomized Controlled Trial. Iranian Red Crescent Medical Journal. 2015;17(11). e20211

[6]. Mohammadi N, Seyedalshohadaei M, Ghasemi nezhad seyede T, Haghani H. Relationship between pressure ulcer, oxygenation and hemodynamic status in mechanically ventilated patients in intensive care units. Thesis for a master's degree in Critical care nursing 2013.

[7]. Setayeshrad S, sadat Ayoubi S, Mehrbakhsh Z, Safarian M, Norouzy A. Assessment of nutritional status, food intake and serum albumin level in patients with pressure ulcers in Qaem training hospital of Mashhad - Iran. Medical Journal of Mashhad University of Medical Sciences. 2015; 58(2): 80-7.

[8]. Burk RS, Grap MJ. Backrest position in prevention of pressure ulcers and ventilator-associated pneumonia: conflicting recommendations. Heart \& Lung: The Journal of Acute and Critical Care. 2012; 41(6): 536-45.

[9]. Borghardt AT, Prado TNd, Araújo TMd, Rogenski NMB, Bringuente MEdO. Evaluation of the pressure ulcers risk scales with critically ill patients: a prospective cohort study. Revista Latino-Americana De Enfermagem. 2015; 23(1): 28-35.

[10]. Hause A. Wireless Pressure Ulcer Prevention Device: Worcester Polytechnic Institute; 2012.

[11]. Michel JM, Willebois S, Ribinik P, Barrois B, Colin D, Passadori Y. As of 2012, what are the key predictive risk factors for pressure ulcers? Developing French guidelines for clinical practice. Annals of Physical and Rehabilitation Medicine. 2012; 55(7): 454-65.

[12]. Källman U. Evaluation of Repositioning in Pressure Ulcer Prevention. Linköping University Medical Dissertations. 2015( 1455 ): 1-89.

[13]. Mehrabani M, Hosseini MA, Nourozi Tabrizi K, Karimloo M. Comparison of Honey dressing with Hydrocolloid dressing effects on pressure ulcer healing of ICU hospitalized patients. Journal of Health Promotion Management. 2012; 1(3): 37-45.

[14]. Benoit Jr, Richard A. Risk factors for pressure ulcer development in critically ill patients. Diss. Vanderbilt University. 2013.

[15]. Šáteková L \& Žiaková K. Validity of pressure ulcer risk assessment scales: review. Central Nursing and Midwifery. 2014; 5(2): 8592.

[16]. Apostolopoulou E, Tselebis A, Terzis K, Kamarinou E, Lambropoulos I, Kalliakmanis A. Pressure ulcer incidence and risk factors in ventilated intensive care patients. Health Science Journal. 2014; 8(3): 333-42.

[17]. Bours GJ, De Laat E, Halfens RJ, Lubbers M. Prevalence, risk factors and prevention of pressure ulcers in Dutch intensive care units; Results of a cross-sectional survey. Intensive Care Med. 2001; 27(10): 1599-605.

[18]. Xakellis GC, Frantz R, Lewis A. Cost of pressure ulcer prevention in long-term care. J Am Geriatr Soc. 1995; 43(5): 496-501.

[19]. Walton-Geer PS. Prevention of pressure ulcers in the surgical patient. AORN J. 2009; 89(3): 538-48.

[20]. O'Brien DD, Shanks AM, Talsma A, Brenner PS, Ramachandran SK. Intraoperative Risk Factors Associated With Postoperative Pressure Ulcers in Critically Ill Patients: A Retrospective Observational Study. Critical Care Medicine. 2014; 42(1): 40-7.

[21]. Vollman KM. Hemodynamic instability: is it really a barrier to turning critically ill patients? Critical care nurse. 2012; 32(1):70-5.

[22]. Mohammadi N, Ghaseminezhad S, Seyedalshohadaei M, Haghani H, Yekaninezhad M. The role of hemodynamic status and the level of oxygenation in the development of pressure ulcer in mechanically ventilated patients. Iran Journal of Nursing. 2013; 25(80): 56-64.

[23]. Nagaya S, Fujimoto E, Kobayashi H. Assessment of Blood Pressure for Determining the Time to Perform First Postural Change in Patients after Cardiac Surgery in the Intensive Care Unit. J Nurs Care. 2014; 3(177): 2167-1168. 
[24]. Alizadeh Ghavidel A, Bashavard S, Bakhshandeh Abkenar H, Payghambari MM. Incidence rate of pressure sores after cardiac surgery during hospitalization and its relevant factors. Razi Journal of Medical Sciences. 2012; 19(102): 18-29.

[25]. Soozani A, Khosravi A, Pourheydari M, Montazeri A. Using Braden and Waterlow Scales to Predict Pressure Ulcer: A Comparative Study. Knowledge \& Health 2011; 5(4): 43-8.

[26]. Man Sp, Au-Yeung Tw. Hypotension is a risk factor for new pressure ulcer occurrence in older patients after admission to an acute hospital. Journal of the American Medical Directors Association. 2013; 14(8): 627- e1.

[27]. Falahinia GhH, Vali Kian M, Shamsaei F, Salavati M, Soltanian AR. Survey of risk factors for development of pressure ulcers in patients with brain - Spinal injuries at home. Scientific Journal of Hamadan Nursing \& Midwifery Faculty. 2013; $21(2)$ : 47-57.

[28]. Bly D, Schallom M, Sona C, Klinkenberg D. A Model of Pressure, Oxygenation, and Perfusion Risk Factors for Pressure Ulcers in the Intensive Care Unit. American Journal of Critical Care. 2016; 25(2): 156-64.

[29]. Terekeci H, Kucukardali Y, Top C, Onem Y, Celik S, Öktenli Ç. Risk assessment study of the pressure ulcers in intensive care unit patients. European Journal of Internal Medicine. 2009; 20(4): 394-7.

[30]. Pender LR, Frazier SK. The relationship between dermal pressure ulcers, oxygenation and perfusion in mechanically ventilated patients. Intensive \& Critical Care Nursing. 2005; 21(1): 29-38.

[31]. Cuddigan J, Frantz RA. Systemic Hemodynamic and Oxygenation Risk Factors for Pressure Ulcer Development in Critical Care. Conference Year: 2005. http://hdl.handle.net/10755/159327.

Table 1: Comparison between the mean and standard deviation of the mean systolic blood pressure, diastolic and mean arterial significant in two groups based on the day of admission

\begin{tabular}{|l|l|l|l|l|l|}
\hline Variable & Hospitalization days & without ulcer & with ulcer & Difference & P value for t-test \\
\hline systolic BP & First & $122.46 \pm 10.25$ & $122.76 \pm 13.26$ & 0.3 & 0.99 \\
\hline diastolic BP & First & $77.67 \pm 8.57$ & $73.42 \pm 9.37$ & 4.25 & 0.04 \\
\hline mean arterial BP & First & $92.52 \pm 8.20$ & $89.89 \pm 9.90$ & 2.63 & 0.20 \\
\hline systolic BP & second & $118.18 \pm 12.71$ & $122.46 \pm 15.20$ & 3.28 & 0.30 \\
\hline diastolic BP & second & $75.58 \pm 9.42$ & $74.58 \pm 9.11$ & 1 & 0.64 \\
\hline mean arterial BP & second & $90.20 \pm 10.04$ & $90.41 \pm 10.38$ & 0.21 & 0.92 \\
\hline systolic BP & Third & $121.87 \pm 14.85$ & $121.74 \pm 13.23$ & 0.13 & 0.97 \\
\hline diastolic BP & Third & $78.40 \pm 10.10$ & $76.27 \pm 9.80$ & 2.13 & 0.47 \\
\hline mean arterial BP & Third & $92.88 \pm 10.74$ & $91.48 \pm 9.75$ & 1.4 & 0.65 \\
\hline systolic BP & Fourth & $110.46 \pm 9.77$ & $115.21 \pm 9.69$ & 4.75 & 0.30 \\
\hline diastolic BP & Fourth & $72.19 \pm 7.32$ & $72.33 \pm 9.75$ & 0.14 & 0.97 \\
\hline mean arterial BP & Fourth & $85.11 \pm 6.98$ & $86.62 \pm 9.29$ & 1.51 & 0.70 \\
\hline
\end{tabular}

Table 2: Comparison of mean and standard deviation of central venous blood pressure in both groups significantly on the day of admission

\begin{tabular}{|l|l|l|l|l|l|}
\hline Variable & Hospitalization days & without ulcer & with ulcer & Difference & P value for t-test \\
\hline CVP & First & $10.72 \pm 2.26$ & $11.74 \pm 3.88$ & 1.02 & 0.13 \\
\cline { 2 - 6 } & second & $11.05 \pm 3.65$ & $15.52 \pm 2.70$ & 4.47 & 0.01 \\
\hline
\end{tabular}

Table 3: Compare the mean and standard deviation of the mean heart rate in both groups significantly on the day of admission

\begin{tabular}{|l|l|l|l|l|l|}
\hline Variable & Hospitalization days & without ulcer & with ulcer & Difference & P value for t-test \\
\hline \multirow{4}{*}{ Heart rate } & First & $86.30 \pm 11.36$ & $90.24 \pm 12.43$ & 3.94 & 0.15 \\
\cline { 2 - 6 } & second & $85.50 \pm 12.78$ & $91.71 \pm 12.91$ & 6.21 & 0.04 \\
\cline { 2 - 6 } & Third & $86.93 \pm 15.23$ & $89.69 \pm 11.91$ & 2.76 & 0.51 \\
\cline { 2 - 6 } & Fourth & $91.13 \pm 8.38$ & $87.08 \pm 15.09$ & 4.05 & 0.50 \\
\hline
\end{tabular}

